\title{
Respiratory fluoroquinolones for the treatment of community-acquired pneumonia: a meta-analysis of randomized controlled trials
}

\author{
Konstantinos Z. Vardakas MD, Ilias I. Siempos MD, Alexandros Grammatikos MD, \\ Zoe Athanassa MD, loanna P. Korbila MD, Matthew E. Falagas MD DSc
}

\section{ABSTRACT}

Background: We investigated whether the use of respiratory fluoroquinolones was associated with better clinical outcomes compared with the use of macrolides and $\beta$ lactams among adults with pneumonia.

Methods: We searched PubMed, Current Contents, Scopus, EMBASE, ClinicalTrials.gov and Cochrane with no language restrictions. Two reviewers independently extracted data from published trials that compared fluoroquinolones (levofloxacin, moxifloxacin, gemifloxacin) with macrolides or $\beta$-lactams or both. A meta-analysis was performed with the clinical outcomes of mortality, treatment success and adverse outcomes.

Results: We included 23 trials in our meta-analysis. There was no difference in mortality among patients who received fluoroquinolones or the comparator antibiotics (OR $0.85,95 \% \mathrm{Cl} 0.65-1.12$ ). Pneumonia resolved in more patients who received fluoroquinolones compared with the comparator antibiotics for the included outcomes in the intention-to-treat population (OR 1.17, 95\% Cl 1.00-1.36), clinically evaluable population (OR 1.26, 95\% Cl 1.06-1.50) and the microbiologically assessed population (OR 1.67, $95 \% \mathrm{Cl} 1.28-2.20)$. Fluoroquinolones were more effective than a combination of $\beta$-lactam and macrolide (OR 1.39, $95 \% \mathrm{Cl} 1.02-1.90)$. They were also more effective for patients with severe pneumonia (OR 1.84, 95\% Cl 1.02-3.29), those who required admission to hospital (OR $=1.30,95 \%$ $\mathrm{Cl}$ 1.04-1.61) and those who required intravenous therapy $(\mathrm{OR}=1.44,15 \% \mathrm{Cl} 1.13-1.85)$. Fluoroquinolones were more effective than $\beta$-lactam and macrolide in open-label trials (OR $=1.35,95 \% \mathrm{Cl} 1.08-1.69)$ but not in blinded randomized controlled trials (OR $=1.13,95 \% \mathrm{Cl} 0.85-1.50)$.

Interpretation: Fluoroquinolones were associated with higher success of treatment for severe forms of pneumonia; however, a benefit in mortality was not evident. A randomized controlled trial that includes patients with severe pneumonia with or without bacteremia is needed.

Une version française de ce résumé est disponible à l'adresse www.cmaj.ca/cgi/content/full/179/12/1269/DC1

CMAJ 2008;179(12):1269-77
$\mathrm{C}$ ommunity-acquired pneumonia is among the leading reasons for hospital admission ${ }^{1}$ and resource consumption..$^{2,3}$ It is the most frequent cause of community-acquired infections among patients admitted to intensive care units. ${ }^{4}$ In addition, it is among the leading causes of death worldwide.

Physicians must choose an optimal therapeutic regimen that eliminates the infection effectively, minimizes the risk of developing drug resistance and does not compromise the safety of the patient. The combination of $\beta$-lactam and macrolide covers the most common possible pathogens involved in the pathogenesis of pneumonia. ${ }^{5}$ More recently, fluoroquinolones with enhanced activity against Streptococcus pneumoniae were introduced in clinical practice. The favourable pharmacokinetic profile of fluoroquinolones allows for once daily administration, often eliminating the need for parenteral treatment. Furthermore, initial treatment with fluoroquinolones was among the predictors of lower treatment failure among patients with pneumonia. ${ }^{6}$

In 2007, the Infectious Diseases Society of America and the American Thoracic Society released new guidelines for the management of care for adult patients with communityacquired pneumonia. ${ }^{7}$ In these guidelines, levofloxacin, gemifloxacin and moxifloxacin were reported to be equally effective as the combination of $\beta$-lactam and macrolide, and were proposed to be the preferred treatment option for patients who require admission to hospital, as well as for patients with comorbidity who receive treatment as outpatients. In addition to being safe, these fluoroquinolones are more effective against the most common types of bacteria responsible for the development of community-acquired pneumonia. ${ }^{7}$ For example, $S$. pneumoniae strains are not fully susceptible to ciprofloxacin. On the other hand, trovafloxacin, clinafloxacin, gatifloxacin and other quinolones are not used because of safety concerns or because they are not widely available. The trials that compared fluoroquinolones with other antibiotics regimens for the treatment of pneumonia were designed on

From the Alfa Institute of Biomedical Sciences (Vardakas, Siempos, Grammatikos, Athanassa, Korbila, Falagas), Athens, Greece; and Tufts University School of Medicine (Falagas), Boston, USA 
the basis of noninferiority (i.e., an antibiotic is equally effective to a comparator), and several were conducted in order to receive approval from the relevant agencies.

We sought to examine whether the use of fluoroquinolones was associated with more advantages or disadvantages than the use of macrolides or $\beta$-lactams in terms of mortality, resolution of pneumonia and adverse effects.

\section{Methods}

\section{Search strategy}

We searched PubMed (1980-2008), Current Contents, Scopus, EMBASE, ClinicalTrials.gov and the Cochrane Central Register of randomized controlled trials using the search terms "community-acquired pneumonia" and "fluoroquinolones," "levofloxacin," "moxifloxacin," "gemifloxacin," "macrolides" or " $\beta$-lactams." Whenever possible, the search was limited to randomized controlled trials. We reviewed the references from the relevant articles, which included review articles. We did not include abstracts from conferences because there is frequently considerable difference between data presented in conference abstracts and the subsequent peerreviewed publications. ${ }^{8,9}$

\section{Study selection}

Two reviewers (I.I.S. and A.G.) independently searched the literature and examined the identified relevant trials for data on effectiveness and toxicity. We considered a trial to be eligible for inclusion in our primary analysis if it compared a fluoroquinolone proposed in the 2007 Infectious Diseases Society of America and the American Thoracic Society guidelines (levofloxacin, gemifloxacin, moxifloxacin) to combination therapy consisting of macrolide and $\beta$-lactam, or to monotherapy (macrolide, ketolide or $\beta$-lactam alone) for the treatment of pneumonia. We excluded trials that compared fluoroquinolones other than those proposed in the guidelines. The inclusion of hospital inpatients was not a prerequisite for eligibility. Trials that included the use of additional antimicrobial agents (mainly those with effectiveness against multi-drug resistant bacteria) were included. We did not set any language restrictions.

\section{Data extraction}

Two reviewers (Z.A. and I.P.K.) independently extracted the relevant data. Any disagreement was resolved by consensus at meetings with all authors. The corresponding authors of the original trials were contacted if additional data were necessary. A quality review of each trial was performed to include details of randomization, generation of random numbers, double-blinding, information on withdrawals, and concealment of allocation. We awarded 1 point for the specification of each criterion to a maximum of 5 points. Trials that scored more than 2 points were considered high quality, and those that scored 2 or fewer points were considered low quality. ${ }^{10}$

\section{Definitions of infections}

We included trials that defined pneumonia according to the following criteria: a baseline chest radiograph that demon- strated new or progressive infiltrates, or consolidation with or without effusion, and 4 of the following signs and symptoms: cough, new or worsened purulent sputum production, rales or signs of pulmonary consolidation or both, dyspnea or hypoxemia or both, fever $\left(\geq 38^{\circ} \mathrm{C}\right)$, respiratory rate greater than 20 breaths/min, systolic hypotension $(<90 \mathrm{~mm} \mathrm{Hg})$, heart rate greater than 120 beats/min; altered mental status, requirement for mechanical ventilation, leukocyte count 10000 cells $/ \mathrm{mm}^{3}$ or greater with $\geq 15 \%$ immature neutrophils, or leukopenia (leukocyte count $\leq 4500$ cells $/ \mathrm{mm}^{3}$ ). For the purpose of our meta-analysis, we considered the severity of pneumonia according to the definitions provided in the individual trials.

We defined bacteremic pneumonia as the presence of S. pneumoniae or another pathogen commonly implicated in the development of pneumonia in 1 or more blood samples, and a clinical profile compatible with a diagnosis of pneumonia.

\section{Outcomes}

Our primary outcome was all-cause mortality in the intentionto-treat population during the study period (e.g., during treatment and follow-up period). Patients who received at least 1 dose of a study medication were included in the intentionto-treat analysis. We also assessed mortality in subgroups of patients with severe pneumonia and bacteremia.

Treatment success ("cure" was defined as resolution of all symptoms and signs of infections; "improvement" was defined as resolution of 2 or more of the baseline symptoms or signs of infections) and adverse outcomes probably or possibly related to the study regimens were considered as secondary outcome measures. Treatment success was assessed in the intention-to-treat population and in the clinically evaluable population (patients who completed the course of treatment and for whom outcome could be assessed at the end of each trial). We also assessed treatment success separately for patients with severe, moderate and mild pneumonia, bacteremic pneumonia, patients who received initial intravenous or oral therapy, those who required hospital admission, those who received levofloxacin, moxifloxacin or gemifloxacin, those who received monotherapy or combination therapy with $\beta$-lactam and macrolide, and patients in the microbiologically evaluable population (patients in the clinically evaluable population with microbiologically confirmed pneumonia) at the testof-cure visit (i.e., when the clinical outcome was assessed) as defined in each trial. We also assessed adverse outcomes probably or possibly related to the study drugs and the duration of stay in hospital.

We performed sensitivity analyses to assess the robustness of the trial findings according to 2 different aspects of the trials methodology: high score on the modified Jadad scale $(>2)$ and use of double blinding. We also performed additional analyses based on whether the study was industry or nonindustry funded.

\section{Data analysis and statistical methods}

We assessed heterogeneity between trials using the $\chi^{2}$ and $I^{2}$ tests. In the analysis of heterogeneity, we considered a $p$ value lower than 0.10 to be statistically significant. We assessed 
publication bias using the funnel plot method and Egger's test. ${ }^{11}$ We calculated pooled odds ratios (OR) and 95\% confidence intervals (CI) for all primary and secondary outcomes using both the Mantel-Haenszel fixed effects ${ }^{12}$ and the DerSimonian-Laird random-effects models. ${ }^{13}$ For all analyses, the results from the fixed-effects model are presented only when there was no heterogeneity between trials; otherwise, the results from the random-effects model are presented.

\section{Results}

\section{Included trials}

We identified a total of 1365 articles that reported on pneumonia treated with fluoroquinolones, $\beta$-lactams and macrolides. Of these, 1086 were excluded because they were not randomized controlled trials. An additional 256 trials were excluded because of reasons shown in Figure 1. Thus, we included 23 trials (which included 7885 patients) in our meta-analyses. ${ }^{14-36}$ The 2 reviewers had initial agreement on 943/1058 (89\%) entries for methodology and outcomes (kappa statistic $=0.78)$.

We contacted the corresponding authors of 19 studies. Of these, 2 authors provided the requested data; 5 additional authors reported that the requested data were not available. We did not find publication bias in the performed analyses. Appendix 1 (available online at www.cmaj.ca/cgi/content/full /179/12/1269/DC2) presents the outcomes of the trials included in the meta-analysis.

The main characteristics of the analyzed trials are shown in Appendix 2 (available at www.cmaj.ca/cgi/content /full/179/12/1269/DC2) and Appendix 3 (available at www.cmaj.ca/cgi/content/full/179/12/1269/DC2). Of the included trials, 8 trials included only patients with severe or moderate-to-severe pneumonia. ${ }^{16,18,19,21,22,28,30,36} \mathrm{Six}$ additional trials enrolled patients with severe pneumonia; ${ }^{17,26,27,29,33,34}$ however, the majority of enrolled patients had mild to moderate pneumonia. The guidelines of the American Thoracic Society $^{37}$ and Pneumonia Severity Index score class IV and $\mathrm{V}^{38}$ were mainly used for assessment of severe pneumonia. Criteria for hospital admission varied between trials, and the need for initial intravenous treatment was the main reason for admission to hospital. Five trials included outpatients. ${ }^{15,20,23,24,33}$ Both intravenous and oral administration were used depending on the severity of pneumonia and the ability of patients to receive medications orally.

For all included trials, we excluded patients from the effectiveness analysis if they had received any antibiotics for treatment of pneumonia in the 24-48 hours before enrollment, unless the isolated pathogen was resistant to these antibiotics or treatment had failed (according to the opinion of the investigators). No data were available from the trials about the management of pneumonia caused by microorganisms that may have been resistant to the study medication.

Details about drug resistance patterns were reported in 13 trials. ${ }^{14-18,20,21,26,27,29-32}$ Isolates recovered from all patients enrolled in the studies were tested for resistance against the study antibiotics and for penicillin resistance. Only one $S$. aureus isolate resistant to levofloxacin was found in these
13 studies. S. pneumoniae strains resistant to the comparator antibiotics were more commonly found (1\%-33\%). Resistance was more prominent among macrolides $(8 \%-33 \%$ for all isolates) than among $\beta$-lactams $(0 \%-11.5 \%$ for all isolates). Intermediate resistance to penicillin was a more common finding ( $8 \%-29 \%$ for all isolates).

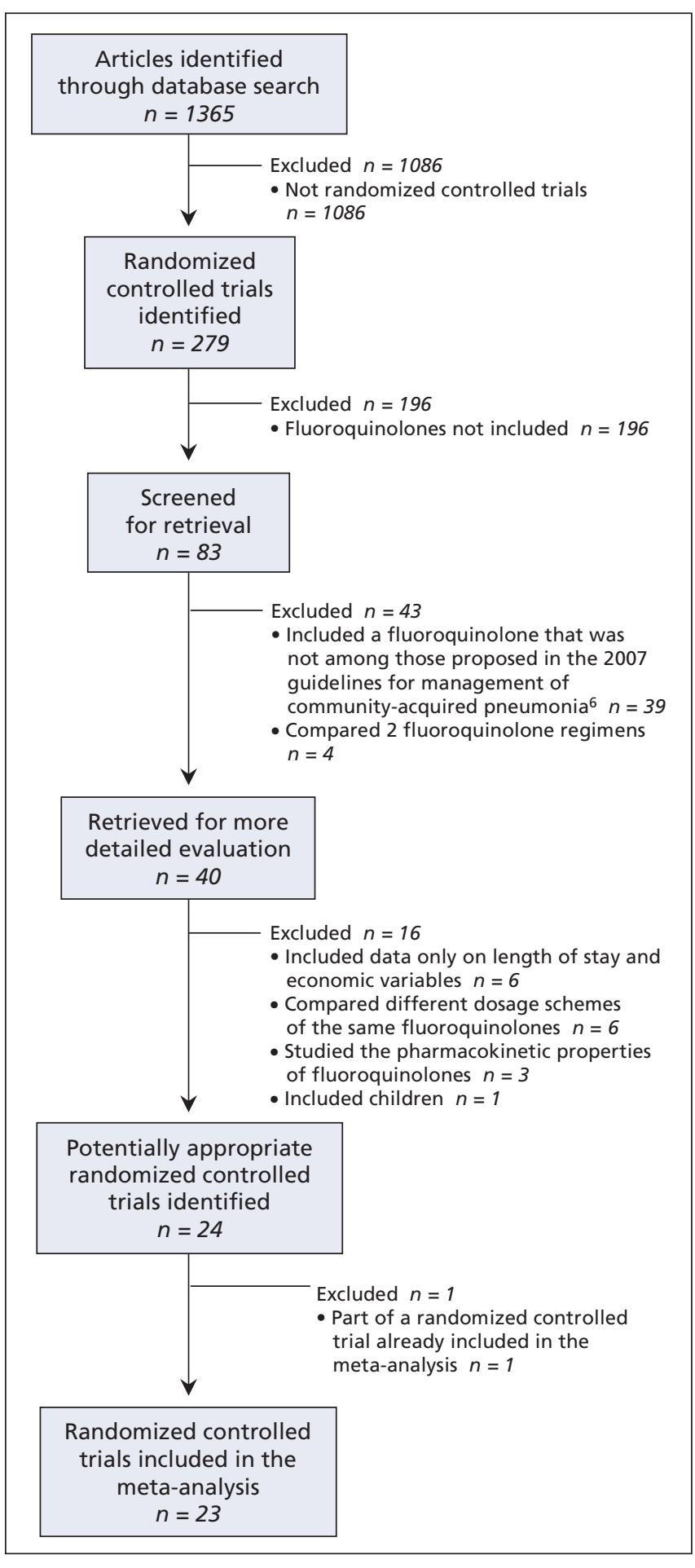

Figure 1: Retrieval and selection of randomized controlled trials for inclusion in the meta-analysis of the effectiveness of fluoroquinolones for the treatment of pneumonia. 


\section{Mortality}

Data about mortality in the intention-to-treat population were provided in 18 trials $^{14-19,21,22,24,26,27,29-34,36}$ and ranged from $0 \%$ to $7 \%$ (mean $2.7 \%$ ) in the fluoroquinolone groups and from $0.5 \%$ to $8 \%$ (mean $3.4 \%$ ) in the comparator groups. Mortality rates were not different between the 2 groups of patients (Figure 2, OR 0.85, 95\% CI 0.65-1.12). Data about mortality among patients with severe pneumonia were only available for 1 trial, ${ }^{16}$ and data about mortality of patients with bacteremic pneumonia were available for 2 trials; ${ }^{16,31}$ thus, we did not perform a combined analysis. Mortality rates were not different when we excluded trials that included outpatients (OR 0.92, 95\% CI 0.68-1.24).

\section{Treatment success}

Data about treatment success in the intention-to-treat population were available for 15 trials. ${ }^{14,15,19-21,26-28,31-36}$ Overall, treatment with fluoroquinolones was successful for $84.2 \%$ of patients. Treatment with comparator antibiotics was successful for $82.2 \%$ of patients. Pneumonia was cured or improved for significantly more patients in the fluoroquinolones group than in the comparison group (Figure 3, OR 1.17, 95\% CI 1.00-1.36).

All trials provided data about treatment success in the clinically evaluable population. ${ }^{14-36}$ The effectiveness of both antibiotic regimens was high (fluoroquinolones 91.9\%, comparator antibiotics $89.7 \%$ ). However, our meta-analysis showed that fluoroquinolones were more effective than the compara- tors antibiotics for the treatment of pneumonia (OR 1.26, 95\% CI 1.06-1.50). Fluoroquinolones were also more effective than combination therapy (OR 1.39, 95\% CI 1.02-1.90). However, there was no difference in treatment success when fluoroquinolones were compared with $\beta$-lactam or macrolide monotherapy (OR 1.19, 95\% CI 0.93-1.50). In the analysis that included only trials not funded by pharmaceutical companies, fluoroquinolones were more effective than the comparator antibiotics (OR 1.86, 95\% CI 1.26-2.75) (Appendix 4: available at www.cmaj.ca/cgi/content/full/179/12/1269 /DC2). ${ }^{18,22,25,27,31,35}$ However, they were not more effective in the analysis that included trials funded by pharmaceutical companies (OR 1.13, 95\% CI 0.93-1.38). ${ }^{14-17,19-21,23,24,26,28-30,32-34,36}$

Twelve trials included patients with severe pneumonia; of these, 7 reported data from this population. ${ }^{16,19,26,27,29,33,34}$ In this subgroup, fluoroquinolones were more effective than the comparator antibiotics (OR 1.84, 95\% CI 1.02-3.29). Data about patients with severe pneumonia who received combination therapy or monotherapy were not provided separately. However, fluoroquinolones were not more effective than comparator antibiotics for treatment of mild to moderate pneumonia (OR 1.22, 95\% CI 0.98-1.51) $)^{14,15,17,18,20,22-29,31}$ and moderate-to-severe pneumonia (OR 1.43, 95\% CI 1.01-2.04). . $^{16,18,19,21,27,28,30,33,36}$

In 11 trials, fluoroquinolones were administered orally. ${ }^{14-17,20,23,24,27,29,31-33}$ In 3 of these trials, the comparator antibiotics were initially administered intravenously. ${ }^{16,17,29}$ In this subgroup of 11 trials, fluoroquinolones were not more effect-

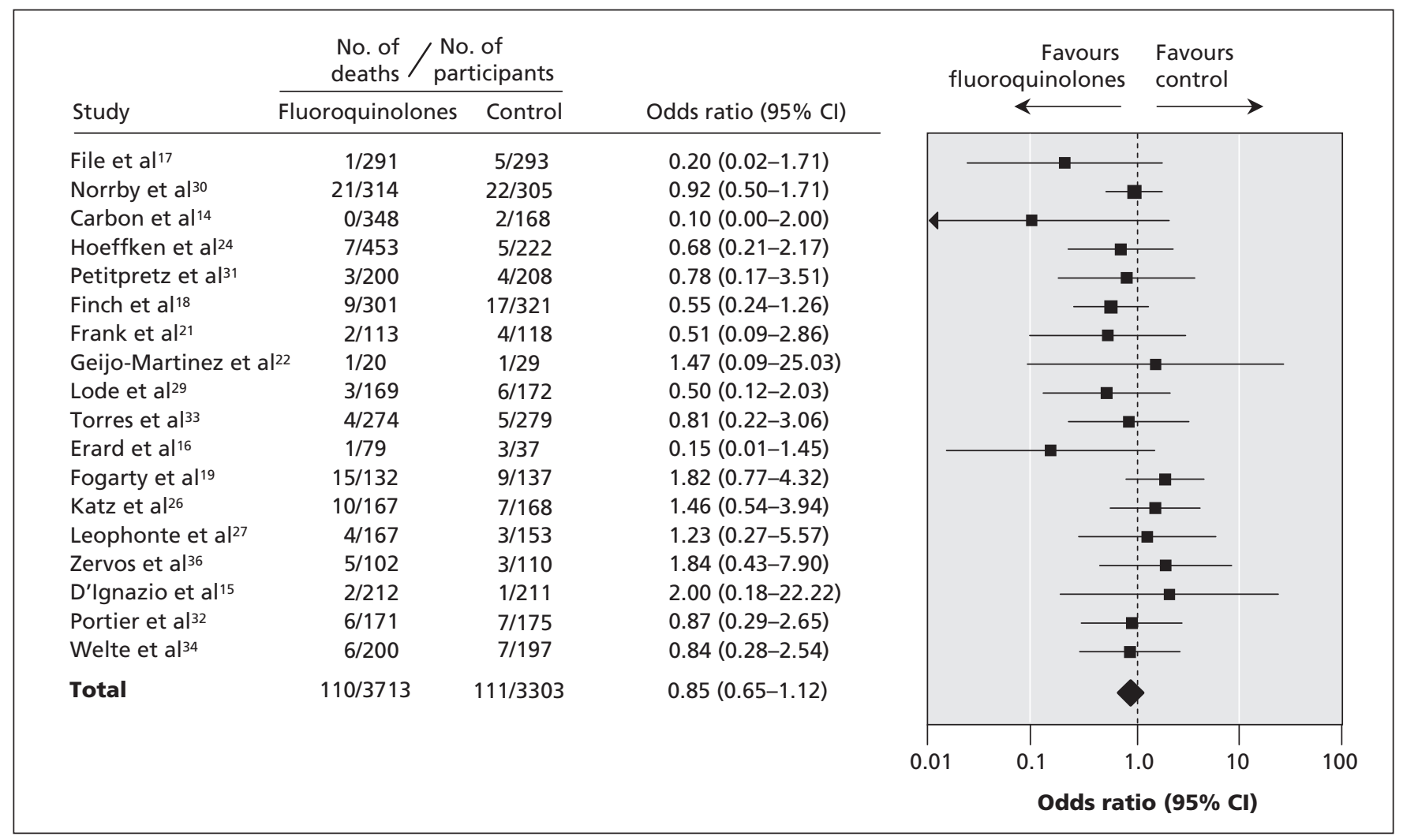

Figure 2: Effect of fluoroquinolones on mortality among patients with pneumonia in the intention-to-treat population. Test for heterogeneity: $\chi^{2}=14.25, p=0.65, I^{2}=0 \%$. Test for overall effect: $z=1.12, p=0.26$. Note: $\mathrm{Cl}=$ confidence interval. 
ive than comparator antibiotics (OR 1.10, 95\% CI 0.85-1.41). In trials that used initial intravenous treatment for either antibiotic regimen, fluoroquinolones were more effective than comparator antibiotics (OR 1.44, 95\% CI 1.13-1.85). ${ }^{17-19,21,22,25,26,28,34-36}$ For trials that included outpatients, there was no benefit with fluoroquinolone treatment (OR 1.06, 95\% CI 0.75-1.50). ${ }^{15,20,23,24,33}$ Among patients in hospital, treatment success was significantly higher with use of fluoroqunilones than with comparator antibiotics (OR 1.30, 95\% CI 1.04-1.61). ${ }^{16,18,19,21,25-32,34-36}$

When we examined the effectiveness of individual fluoroquinolones, we found no difference between levofloxacin and the comparator antibiotics (OR 1.28, 95\% CI 0.97-1.67). ${ }^{14-17,19,21-23,25,28,30,36}$ Similar findings were reported for moxifloxacin (OR 1.22, 95\% CI 0.95-1.55). Levofloxacin was administered to outpatients in 3 trials, ${ }^{14,15,23}$ and it was administered orally in 4 trials. ${ }^{14-16,23}$ Moxifloxacin was administered to outpatients in 2 trials, ${ }^{20,24}$ and it was administered orally in 5 trials..$^{20,24,31-33}$

In the microbiologically evaluable population, fluoroquinolones were more effective than comparator antibiotics (OR 1.67, 95\% CI 1.28-2.20). ${ }^{14,15,17-24,26-32,35}$ For the subgroup of patients with pneumonia due to $S$. pneumoniae, there was no difference in effectiveness (OR 0.72, 95\% CI 0.39-1.33). ${ }^{15,17,19,27-29,31,32,35,36}$ Data about other possible pathogens, including Hemophilus influenzae, S. aureus, Pseudomonas spp and atypical pathogens, were not reported consistently. There was no difference in effectiveness among patients with bacteremic pneumonia (OR 0.84, 95\% CI
$0.37-1.89)^{16,18,20,21,26,27,29,31,32,36}$ or bacteremic pneumonia due to S. pneumoniae (OR 1.30, 95\% CI 0.43-3.95). ${ }^{17-19,21,26,27,29,32,36}$

\section{Duration of hospital stay}

Data about the duration of stay in hospital were available for 9 trials. ${ }^{16,18,22,28-30,33,34,36}$ Because duration was not uniformly presented, we could not perform a pooled analysis. In total, 5 trials ${ }^{16,29,30,33,34}$ provided the median, and 4 trials ${ }^{18,22,28,36}$ provided the mean duration of hospital stay. Patients who received fluoroquinolones stayed in hospital 1-2 days less than those who received comparator antibiotics in the trials that reported the median length of stay. However, there was no difference in the length of stay in trials that reported the mean length of stay.

\section{Quality assessment and sensitivity analyses}

The mean quality score of the included trials was 2.6 (range $1-5)$. The quality of 11 trials was high. ${ }^{14,15,19-21,24,26-28,30,33}$ The quality of the other 12 studies was low. ${ }^{16-18,22,23,25,29,31,32,34-36}$ There was no difference in the effectiveness of fluoroquinolones and comparator antibiotics in the trials that were of high quality (OR 1.22, 95\% CI 0.95-1.57). In the analysis of lowquality trials, fluoroquinolones were associated with significantly improved treatment success (OR 1.30, 95\% CI 1.01-1.67). There was no difference between the antibiotic regimens in sensitivity analyses that included only doubleblinded trials (OR 1.13, 95\% CI 0.85-1.50)..$^{14,15,20,23,24,27,31,33}$ However, there was a significant difference in favour of fluoroquinolones when only open-label trials were included (OR $1.35,95 \%$ CI $1.08-1.69){ }^{13-19,21,22,25,26,28-30,32,34-36}$

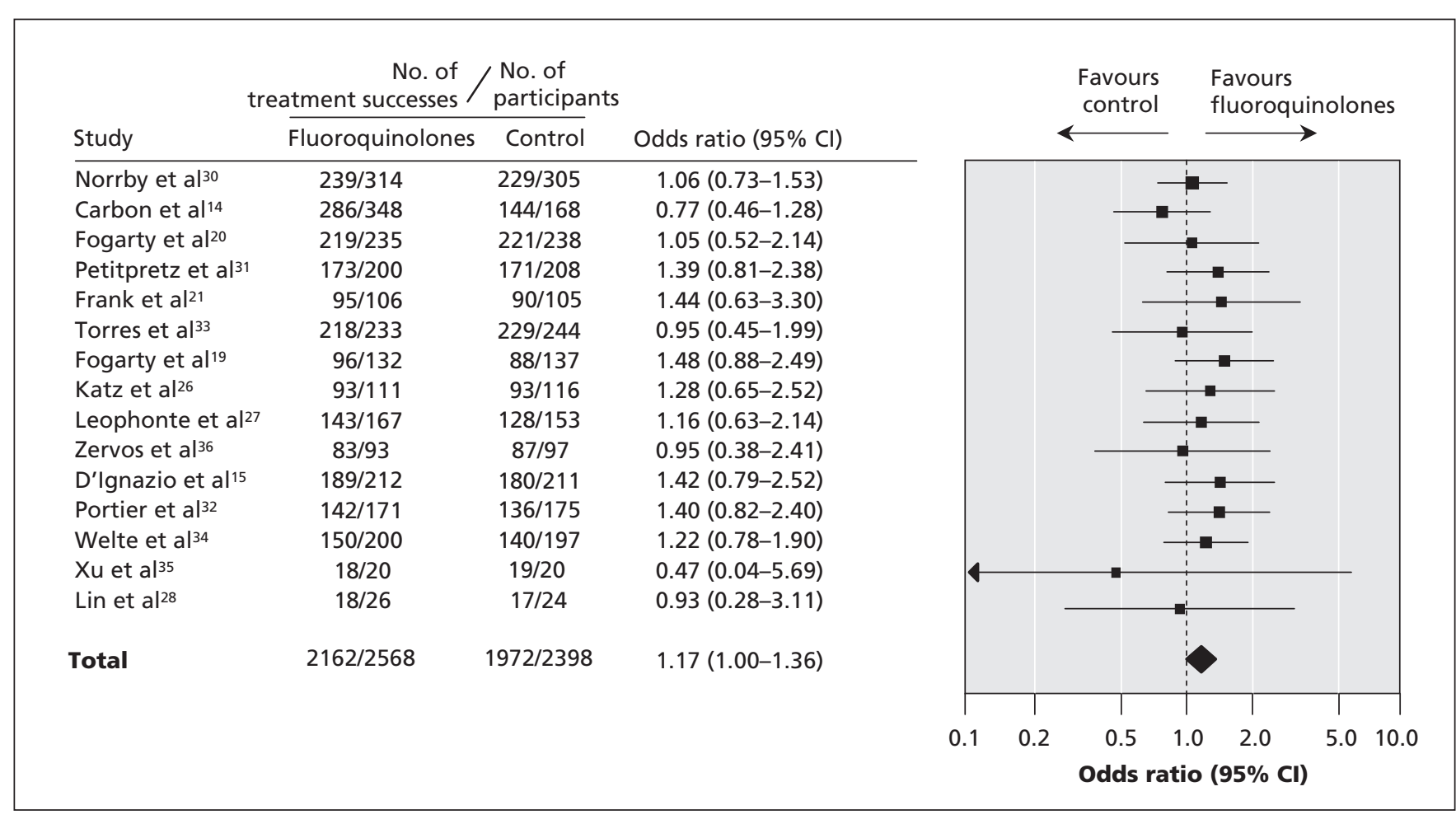

Figure 3: Odds ratios for treatment success with fluoroquinolone therapy among patients with pneumonia for the intention-to-treat and clinically evaluable populations. Test for heterogeneity: $\chi^{2}=6.49, p=0.95, l^{2}=0 \%$. Test for overall effect: $z=1.96, p=0.05$. Note: $\mathrm{Cl}=$ confidence interval. 


\section{Adverse outcomes}

All trials reported data about drug-related adverse outcomes in the intention-to-treat population. The majority of adverse outcomes were mild to moderately severe disturbances of the gastrointestinal tract. The most commonly studied adverse effects were photosensitivity, diarrhea, vomiting and nausea, liver function abnormalities, insomnia, headache and rash (Appendix 5: available at www.cmaj.ca/cgi/content/full/179 /12/1269/DC2). Fluoroquinolones were associated with significant fewer adverse outcomes than comparator antibiotics (OR 0.86, 95\% CI 0.78-0.96) (Figure 4). The percentage of patients who were withdrawn from the trials because of drugrelated adverse outcomes was not different between groups (OR $0.85,95 \%$ CI $0.69-1.06$ ) and was mostly because of gastrointestinal disturbances. Thirteen trials reported data about recurrent infections. ${ }^{14-17,20,24,28-32,34}$ There was no difference between fluoroquinolones and the compared antibiotics for this outcome (OR 0.98, 95\% CI 0.59-1.63).

\section{Interpretation}

We found no differences in mortality between patients with community-acquired pneumonia who received fluoro- quinolones and those who received a combination of $\beta$-lactam and macrolide or either antibiotic alone. In contrast, treatment success in the clinically evaluable and intention-to-treat populations was significantly higher among patients who received fluoroquinolones. This effect was more prominent among patients with severe pneumonia (9\% absolute difference in treatment success). We also found that fluoroquinolones were more effective than the comparator antibiotics when administered in hospital or when initial treatment was administered intravenously. Trials that were not funded by industry showed a significantly higher treatment success among patients who received fluoroquinolones compared to the comparator antibiotics. Fluoroquinolones were also associated with fewer adverse outcomes. However, previous meta-analyses have reported that fluoroquinolones were associated with either more adverse outcomes compared with other antibiotics or no difference in toxicity was reported..$^{39-43}$

The findings of our meta-analysis about overall treatment success were similar to the results of a meta-analysis published in 2002. ${ }^{44}$ However, only 3 of the trials included in that meta-analysis studied the effectiveness of the fluoroquinolones proposed in the 2007 guidelines. In addition, the authors of the 2002 meta-analysis stated that data about mor-

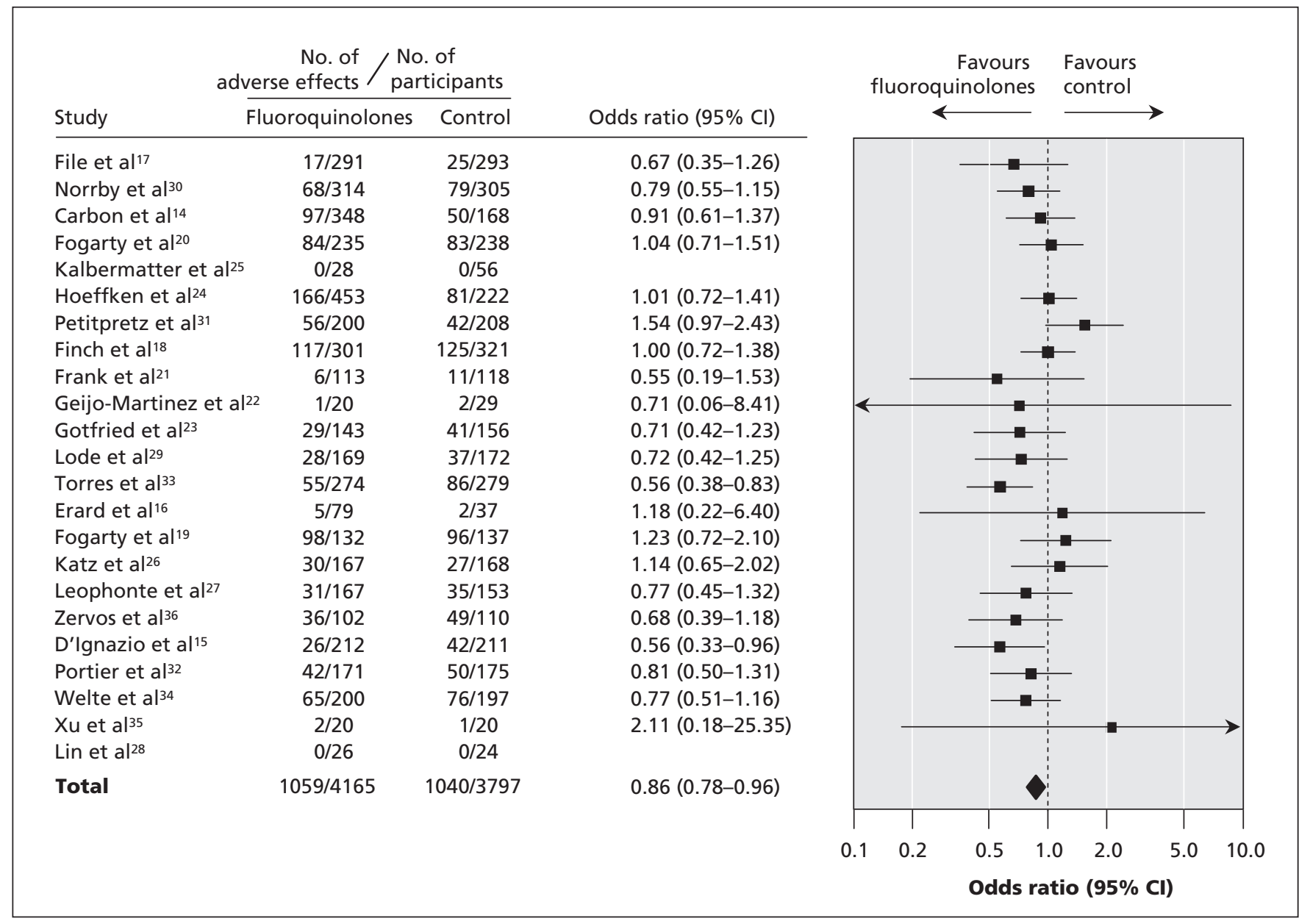

Figure 4: Odds ratios for adverse outcomes after fluoroquinolone therapy among patients with pneumonia in the intention-to-treat population. Test for heterogeneity: $\chi^{2}=22.81, p=0.30, R=12.3 \%$. Test for overall effect: $z=2.72, p=0.007$. Note: $\mathrm{Cl}=$ confidence interval. 
tality were not available. In addition, they did not differentiate between mild, moderate and severe forms of pneumonia, and they did not analyze data about bacteremic pneumonia.

The relatively low mortality reported in the individual trials supports the opinion that the patients enrolled in such trials are not at high risk for death. ${ }^{45}$ In addition, most of the included trials did not provide data about mortality nor were they focused on severe pneumonia. This limited our ability to draw conclusions about the effectiveness of treatment in the most severely ill patients. Two non-randomized trials reported different conclusions; one favoured levofloxacin and one favoured a combination of $\beta$-lactam and macrolide. ${ }^{46,47}$

A possible explanation for the higher treatment success in the fluoroquinolone groups may be because there was zero baseline resistance of the isolated pathogens to fluoroquinolones compared to the higher levels of resistance found for $\beta$-lactams and especially macrolides. However, the trials included in our analysis mainly examined $S$. pneumoniae isolates. The impact of initially appropriate treatment with $\beta$-lactams for the treatment of pneumococcal pneumonia remains questionable. ${ }^{48}$

The Tracking Resistance in the United States Today (TRUST) study reported that although S. pneumoniae isolates were highly resistant to penicillin and azithromycin (15\%-44\% according to the age group of patients from whom the isolates were recovered), resistance to ceftriaxone and amoxicillin-clavulanate was lower (1\%-10\%) and was minimal for levofloxacin $(0 \%-1.3 \%){ }^{49,50}$ Other studies have reported similar findings. ${ }^{51-54}$ In contrast, resistance among $H$. influenzae and Morraxella catarrhalis isolates was high for ampicillin (19\%-98\%) but minimal (0\%-0.2\%) for all other antibiotics. ${ }^{49,50}$ However, resistance to fluoroquinolones may increase in the future, and this may have an impact on treatment outcomes. ${ }^{55}$

Administration of fluoroquinolones was associated with better treatment success among patients with severe pneumonia. The diversity in the definition of severe pneumonia among the individual trials and the use of the Pneumonia Severity Index score as a measure for severe pneumonia may limit this finding. ${ }^{38}$ The use of the CURB-65 (confusion, urea, respiratory rate, blood pressure, age $>65$ ) score may be more appropriate for the assessment of the severity of pneumonia. ${ }^{56}$

Fluoroquinolones were more effective than combination therapy but no differences were seen for monotherapy. This may be because the trials that used combination therapies enrolled mainly patients with more severe forms of pneumonia and, as noted above, fluoroquinolones were more effective than $\beta$-lactams or macrolides in patients with moderate to severe pneumonia. As well, severe pneumonia was an exclusion criterion in the majority of the trials that compared fluoroquinolones with monotherapy.

Patients with bacteremic pneumonia did not seem to benefit more from fluoroquinolones than from $\beta$-lactams or macrolides. We were not able to distinguish between severe and nonsevere bacteremic pneumonia in the included trials. Combination therapy that included macrolides but not fluoroquinolones was associated with lower mortality among patients with bacteremic pneumonia. ${ }^{57-61} \mathrm{~A}$ review of pneumo- coccal bacteremic pneumonia treated with levofloxacin, which also included penicillin-resistant strains, found that $91 \%$ of these patients had a successful clinical response and mortality was lower than $1 \% .^{62}$

We found that orally administered fluoroquinolones were not more effective than orally administered $\beta$-lactams and macrolides. This is in contrast to the favourable pharmacokinetic profile of fluoroquinolones. ${ }^{63,64}$ Oral administration was mainly used for patients with milder forms of pneumonia, for whom the success rate of treatment was expected to be higher. In these studies, levofloxacin dosage was suboptimal (500 mg instead of $750 \mathrm{mg}$ or more as currently proposed), which may have influenced the results. In contrast, fluoroquinolones were more effective among patients who required admission to hospital or intravenous treatment, which indicates that fluoroquinolones might be the preferred antibiotic in this settings. However, the diversity of the criteria used for determining inpatient or intravenous treatment among the trials included in our meta-analysis may limit the generalizability of our findings.

\section{Limitations}

The major limitation of our meta-analysis is the relatively low quality of the included trials. Only 8 of the included trials were double-blinded. We performed a sensitivity analysis that included only trials that were of high quality or that were double-blinded. This analysis showed that fluoroquinolones were not more effective than comparator antibiotics for the treatment of pneumonia. Patients with milder forms of pneumonia were enrolled in these high-quality trials.

Another limitation was the inclusion of only trials that studied the fluoroquinolones included in the Infectious Diseases Society of America 2007 guidelines. $^{7}$ As a result, 39 trials were not included in the final analysis.

Finally, data on adjunctive therapies that may improve outcomes in patients with severe pneumonia (e.g., hydrocortisone, statins, angiotensin-converting-enzyme inhibitors) were not collected. ${ }^{65}$

We did not present data about the total duration of treatment or the duration of intravenous therapy. These outcomes are subject to the individual investigator's assessment of the need for additional antibiotic treatment because the optimal duration of treatment for pneumonia has not been thoroughly evaluated. ${ }^{66}$ Second, in the studies that enrolled patients with mild to moderately severe pneumonia, the duration of treatment was predefined. Third, a recent meta-analysis showed that the duration of treatment is not associated with clinical outcomes among patients with mild to moderately severe pneumonia. ${ }^{67}$

\section{Conclusion}

The results of our meta-analysis suggest that fluoroquinolones may be considered for the treatment of community-acquired pneumonia. In particular, they should be considered for the more severe forms of pneumonia, as well as for patients who require admission to hospital and initial intravenous treatment. We found no differences in mortality between antibiotic regimens; however, data on mortality among patients 
with severe pneumonia were not available in the trials included in the analyses. A well-designed randomized controlled trial including mainly patients with severe communityacquired pneumonia with or without bacteremia is needed. Finally, physicians are reminded that macrolides and $\beta$ lactams are also highly effective for the treatment of pneumonia, especially for the mild to moderate forms of the infection.

This article has been peer reviewed.

Competing interests: None declared.

Contributors: Konstantinos Vardakas and Matthew Falagas conceived and designed the meta-analysis. Konstantinos Vardakas, Ilias Siempos, Alexandros Grammatikos, Zoe Athanassa and Ioanna Korbila contributed to the literature search, study selection and data extraction. Konstantinos Vardakas performed the analysis and wrote the first version of the manuscript. All of the authors made substantial revisions to the manuscript and approved the final version submitted for publication.

Acknowledgements: We thank Dr. Veronique Erard and Dr. Patrick Petitpretz for providing the additional requested data.

Funding: No external funding was received for this research.

\section{REFERENCES}

1. Marston BJ, Plouffe JF, File TM Jr, et al. Incidence of community-acquired pneumonia requiring hospitalization. Results of a population-based active surveillance Study in Ohio. Arch Intern Med 1997;157:1709-18.

2. Guest JF, Morris A. Community-acquired pneumonia: the annual cost to the National Health Service in the United Kingdom. Eur Respir J 1997;10:1530-4.

3. Niederman MS, McCombs JS, Unger AN, et al. The cost of treating communityacquired pneumonia. Clin Ther 1998;20:820-37.

4. Rello J, Catalan M, Diaz E, et al. Associations between empirical antimicrobial therapy at the hospital and mortality in patients with severe community-acquired pneumonia. Intensive Care Med 2002;28:1030-5.

5. Felmingham D, Canton R, Jenkins SG. Regional trends in beta-lactam, macrolide, fluoroquinolone and telithromycin resistance among Streptococcus pneumoniae isolates 2001-2004. J Infect 2007;55:111-8.

6. Menendez R, Torres A, Zalacain R, et al. Neumofail Group. Risk factors of treatment failure in community acquired pneumonia: implications for disease outcome. Thorax 2004;59:960-5.

7. Mandell LA, Wunderink RG, Anzueto A, et al.; Infectious Diseases Society of America. American Thoracic Society. Infectious Diseases Society of America/ American Thoracic Society consensus guidelines on the management of communityacquired pneumonia in adults. Clin Infect Dis 2007;44(Suppl 2):S27-72.

8. Rosmarakis ES, Soteriades ES, Vergidis PI, et al. From conference abstract to full paper: differences between data presented in conferences and journals. FASEB $J$ 2005;19:673-80.

9. Falagas ME, Rosmarakis ES. Clinical decision-making based on findings presented in conference abstracts: Is it safe for our patients? Eur Heart J 2006;27: 2038-9.

10. Moher D, Pham B, Jones A, et al. Does quality of reports of randomised trials affect estimates of intervention efficacy reported in meta-analyses? Lancet 1998; 352:609-13.

11. Egger M, Davey Smith G, Schneider M, et al. Bias in meta-analysis detected by a simple, graphical test. BMJ 1997;315:629-34.

12. Mantel N, Haenszel W. Statistical aspects of the analysis of data from retrospective studies of disease. J Natl Cancer Inst 1959;22:719-48.

13. DerSimonian R, Laird N. Meta-analysis in clinical trials. Control Clin Trials 1986; 7:177-88.

14. Carbon C, Ariza H, Rabie WJ, et al. Comparative study of levofloxacin and amoxycillin/clavulanic acid in adults with mild-to-moderate community-acquired pneumonia. Clin Microbiol Infect 1999;5:724-32.

15. D'Ignazio J, Camere MA, Lewis DE, et al. Novel, single-dose microsphere formulation of azithromycin versus 7-day levofloxacin therapy for treatment of mild to moderate community-acquired pneumonia in adults. Antimicrob Agents Chemother 2005;49:4035-41.

16. Erard V, Lamy O, Bochud PY, et al. Full-course oral levofloxacin for treatment of hospitalized patients with community-acquired pneumonia. Eur J Clin Microbiol Infect Dis 2004;23:82-8.

17. File TM Jr, Segreti J, Dunbar L, et al. A multicenter, randomized study comparing the efficacy and safety of intravenous and/or oral levofloxacin versus ceftriaxone and/or cefuroxime axetil in treatment of adults with community-acquired pneumonia. Antimicrob Agents Chemother 1997;41:1965-72.

18. Finch R, Schurmann D, Collins O, et al. Randomized controlled trial of sequential intravenous (i.v.) and oral moxifloxacin compared with sequential i.v. and oral co- amoxiclav with or without clarithromycin in patients with community-acquired pneumonia requiring initial parenteral treatment. Antimicrob Agents Chemother 2002;46:1746-54

19. Fogarty C, Siami G, Kohler R, et al. Multicenter, open-label, randomized study to compare the safety and efficacy of levofloxacin versus ceftriaxone sodium and erythromycin followed by clarithromycin and amoxicillin-clavulanate in the treatment of serious community-acquired pneumonia in adults. Clin Infect Dis 2004;38(Suppl 1):S16-23.

20. Fogarty C, Grossman C, Williams J, et al. Efficacy and safety of moxifloxacin vs clarithromycin for community-acquired pneumonia. Infect Med 1999;16:748-63.

21. Frank E, Liu J, Kinasewitz G, et al. A multicenter, open-label, randomized comparison of levofloxacin and azithromycin plus ceftriaxone in hospitalized adults with moderate to severe community-acquired pneumonia. Clin Ther 2002;24:1292-308.

22. Geijo Martinez MP, Diaz de Tuesta Chow-Quan AM, Herranz CR, et al. Levofloxacin versus beta-lactamic therapy in community acquired pneumonia that requires hospitalization. Med Interna 2002;19:621-5.

23. Gotfried MH, Dattani D, Riffer E, et al. A controlled, double-blind, multicenter study comparing clarithromycin extended-release tablets and levofloxacin tablets in the treatment of community-acquired pneumonia. Clin Ther 2002;24:736-51.

24. Hoeffken G, Meyer HP, Winter J, et al.; Community-acquired Pneumonia Study Group. The efficacy and safety of two oral moxifloxacin regimens compared to oral clarithromycin in the treatment of community-acquired pneumonia. Respir Med 2001;95:553-64

25. Kalbermatter V, Bagilet D, Diab M, et al. Oral levofloxacin versus intravenous ceftriaxone and amoxicillin/clavulanic acid in the treatment of community-acquired pneumonia that requires hospitalization. Med Clin (Barc) 2000;115:561-3.

26. Katz E, Larsen LS, Fogarty CM, et al. Safety and efficacy of sequential i.v. to p.o. moxifloxacin versus conventional combination therapies for the treatment of community-acquired pneumonia in patients requiring initial i.v. therapy. J Emerg Med 2004;27:395-405.

27. Leophonte P, File T, Feldman C. Gemifloxacin once daily for 7 days compared to amoxicillin/clavulanic acid thrice daily for 10 days for the treatment of communityacquired pneumonia of suspected pneumococcal origin. Respir Med 2004;98:708-20.

28. Lin TY, Lin SM, Chen HC, et al. An open label randomized comparison of levofloxacin and amoxicillin/clavulanate plus clarithromycin for the treatment of hospitalized patients with community-acquired pneumonia. Chang Gung Med J 2007; 30:321-32.

29. Lode H, File TM Jr, Mandell L, et al.; 185 Gemifloxacin Study Group. Oral gemifloxacin versus sequential therapy with intravenous ceftriaxone/oral cefuroxime with or without a macrolide in the treatment of patients hospitalized with community-acquired pneumonia: a randomized, open-label, multicenter study of clinical efficacy and tolerability. Clin Ther 2002;24:1915-36.

30. Norrby SR, Petermann W, Willcox PA, et al. A comparative study of levofloxacin and ceftriaxone in the treatment of hospitalized patients with pneumonia. Scand J Infect Dis 1998;30:397-404

31. Petitpretz P, Arvis P, Marel M, et al:; CAP5 Moxifloxacin Study Group. Oral moxifloxacin vs high-dosage amoxicillin in the treatment of mild-to-moderate, communityacquired, suspected pneumococcal pneumonia in adults. Chest 2001;119:185-95.

32. Portier H, Brambilla C, Garre M, et al. Moxifloxacin monotherapy compared to amoxicillin-clavulanate plus roxithromycin for nonsevere community-acquired pneumonia in adults with risk factors. Eur J Clin Microbiol Infect Dis 2005;24:367-76.

33. Torres A, Muir JF, Corris P, et al. Effectiveness of oral moxifloxacin in standard first-line therapy in community-acquired pneumonia. Eur Respir J 2003;21:135-43.

34. Welte T, Petermann W, Schurmann D, et al.; MOXIRAPID Study Group. Treatment with sequential intravenous or oral moxifloxacin was associated with faster clinical improvement than was standard therapy for hospitalized patients with community-acquired pneumonia who received initial parenteral therapy. Clin Infect Dis 2005;41:1697-705.

35. Xu S, Xiong S, Xu Y, et al. Efficacy and safety of intravenous moxifloxacin versus cefoperazone with azithromycin in the treatment of community acquired pneumonia. J Huazhong Univ Sci Technolog Med Sci 2006;26:421-4.

36. Zervos M, Mandell LA, Vrooman PS, et al. Comparative efficacies and tolerabilities of intravenous azithromycin plus ceftriaxone and intravenous levofloxacin with step-down oral therapy for hospitalized patients with moderate to severe community-acquired pneumonia. Treat Respir Med 2004;3:329-36.

37. Niederman MS, Bass JB Jr, Campbell GD, et al. Guidelines for the initial management of adults with community-acquired pneumonia: diagnosis, assessment of severity, and initial antimicrobial therapy. American Thoracic Society. Medical Section of the American Lung Association. Am Rev Respir Dis 1993;148:1418-26.

38. Fine MJ, Auble TE, Yealy DM, et al. A prediction rule to identify low-risk patients with community-acquired pneumonia. N Engl J Med 1997;336:243-50.

39. Shorr AF, Susla GB, Kollef MH. Quinolones for treatment of nosocomial pneumonia: a meta-analysis. Clin Infect Dis 2005;40(Suppl 2):S115-22.

40. Falagas ME, Matthaiou DK, Vardakas KZ. Fluoroquinolones vs beta-lactams for empirical treatment of immunocompetent patients with skin and soft tissue infections: a meta-analysis of randomized controlled trials. Mayo Clin Proc 2006;81:1553-66.

41. Siempos II, Vardakas KZ, Manta KG, et al. Carbapenems for the treatment of immunocompetent adult patients with nosocomial pneumonia. Eur Respir J 2007;29: $548-60$.

42. Matthaiou DK, Peppas G, Bliziotis IA, et al. Ciprofloxacin/metronidazole versus beta-lactam-based treatment of intra-abdominal infections: a meta-analysis of comparative trials. Int J Antimicrob Agents 2006;28:159-65.

43. Bliziotis IA, Michalopoulos A, Kasiakou SK, et al. Ciprofloxacin vs an aminoglycoside in combination with a beta-lactam for the treatment of febrile neutropenia: a meta-analysis of randomized controlled trials. Mayo Clin Proc 2005;80:1146-56. 
44. Salkind AR, Cuddy PG, Foxworth JW. Fluoroquinolone treatment of communityacquired pneumonia: a meta-analysis. Ann Pharmacother 2002;36:1938-43.

45. Shefet D, Robenshtock E, Paul M, et al. Empiric antibiotic coverage of atypical pathogens for community acquired pneumonia in hospitalized adults. Arch Intern Med 2005;165:1992-2000.

46. Querol-Ribelles JM, Tenias JM, Querol-Borras JM, et al. Levofloxacin versus ceftriaxone plus clarithromycin in the treatment of adults with community-acquired pneumonia requiring hospitalization. Int J Antimicrob Agents 2005;25:75-83.

47. Lodise TP, Kwa A, Cosler L, et al. Comparison of $\beta$-lactam and macrolide combination therapy versus fluoroquinolone monotherapy in hospitalized veterans affairs patients with community-acquired pneumonia. Antimicrob Agents Chemother 2007;51:3977-82.

48. Falagas ME, Siempos II, Bliziotis IA, et al. Impact of initial discordant treatment with beta-lactam antibiotics on clinical outcomes in adults with pneumococcal pneumonia: a systematic review. Mayo Clin Proc 2006;81:1567-74.

49. Karlowsky JA, Thornsberry C, Critchley IA, et al. Susceptibilities to levofloxacin in Streptococcus pneumoniae, Haemophilus influenzae, and Moraxella catarrhalis clinical isolates from children: results from 2000-2001 and 2001-2002 TRUST studies in the United States. Antimicrob Agents Chemother 2003;47:1790-7.

50. Karlowsky JA, Thornsberry C, Jones ME, et al.; TRUST Surveillance Program. Factors associated with relative rates of antimicrobial resistance among Streptococcus pneumoniae in the United States: results from the TRUST Surveillance Program (1998-2002). Clin Infect Dis 2003;36:963-70.

51. Morrissey I, Robbins M, Viljoen L, et al. Antimicrobial susceptibility of community-acquired respiratory tract pathogens in the UK during 2002/3 determined locally and centrally by BSAC methods. J Antimicrob Chemother 2005;55:200-8.

52. Jenkins SG, Brown SD, Farrell DJ. Trends in antibacterial resistance among Streptococcus pneumoniae isolated in the USA: update from the PROTEKT US Years 1-4. Ann Clin Microbiol Antimicrob 2008;7:1.

53. Adam HJ, Schurek KN, DeCorby MR, et al. Comparative in vitro activity of PGE 9262932 and fluoroquinolones against Canadian clinical Streptococcus pneumoniae isolates, including molecularly characterized ciprofloxacin-resistant isolates. $J$ Antimicrob Chemother 2006;58:202-4.

54. Doern GV, Richter SS, Miller A, et al. Antimicrobial resistance among Streptococcus pneumoniae in the United States: Have we begun to turn the corner on Rresistance to certain antimicrobial classes? Clin Infect Dis 2005;41:139-48.

55. Adam HJ, Schurek KN, Nichol KN, et al. Molecular characterization of increasing fluoroquinolone resistance in Streptococcus pneumoniae isolates in Canada, 1997 to 2005. Antimicrob Agents Chemother 2007;51:198-207.

56. Lim WS, van der Eerden MM, Laing R, et al. Defining community acquired pneumonia severity on presentation to hospital: an international derivation and validation study. Thorax 2003;58:377-82.

57. Martinez JA, Horcajada JP, Almela M, et al. Addition of a macrolide to a $\beta$-lactambased empirical antibiotic regimen is associated in lower in-hospital mortality for patients with bacteremic pneumococcal pneumonia. Clin Infect Dis 2003;36:389-95.

58. Baddour LM, Yu VL, Klugman KP, et al. Combination antibiotic therapy lowers mortality among severely ill patients with pneumococcal bacteremia. Am J Respir Crit Care Med 2004;170:440-4.

59. Waterer GW, Somes GW, Wunderink RG. Monotherapy may be suboptimal for severe bacteremic pneumococcal pneumonia. Arch Intern Med 2001;161:1837-42.

60. Metersky ML, Ma A, Houck PM, et al. Antibiotics for bacteremic pneumonia: improved outcomes with macrolides but not fluoroquinolones. Chest 2007;131:466-73.

61. Vardakas KZ, Falagas ME. Interpreting the findings of a cohort study in bacteremic patients with community acquired pneumonia. Chest 2007;132:1715-6.

62. Kahn JB, Bahal N, Wiesinger BA, et al. Cumulative clinical trial experience with levofloxacin for patients with community-acquired pneumonia-associated pneumococcal bacteremia. Clin Infect Dis 2004;38(Suppl 1):S34-42.

63. Chien SC, Rogge MC, Gisclon LG, et al. Pharmacokinetic profile of levofloxacin following once-daily 500-milligram oral or intravenous doses. Antimicrob Agents Chemother 1997;41:2256-60.

64. Sullivan JT, Woodruff M, Lettieri J, et al. Pharmacokinetics of a once-daily oral dose of moxifloxacin (Bay 12-8039), a new enantiomerically pure 8-methoxy quinolone. Antimicrob Agents Chemother 1999;43:2793-7.

65. Siempos II, Vardakas KZ, Kopterides $\mathrm{P}$, et al.Adjunctive therapies for communityacquired pneumonia: a systematic review. J Antimicrob Chemother. 2008;62:661-8.

66. File TM Jr. Current challenges in the treatment of community-acquired pneumonia. Clin Infect Dis 2004;38(Suppl 1):S1-4.

67. Li JZ, Winston LG, Moore DH, et al. Efficacy of short-course antibiotic regimen for community-acquired pneumonia: a meta-analysis. Am J Med 2007;120:783-90.

Correspondence to: Dr. Matthew E. Falagas, 9 Neapoleos St. 15123 Marousi, Greece; fax 30-210-68 39 605;

m.falagas@aibs.gr 